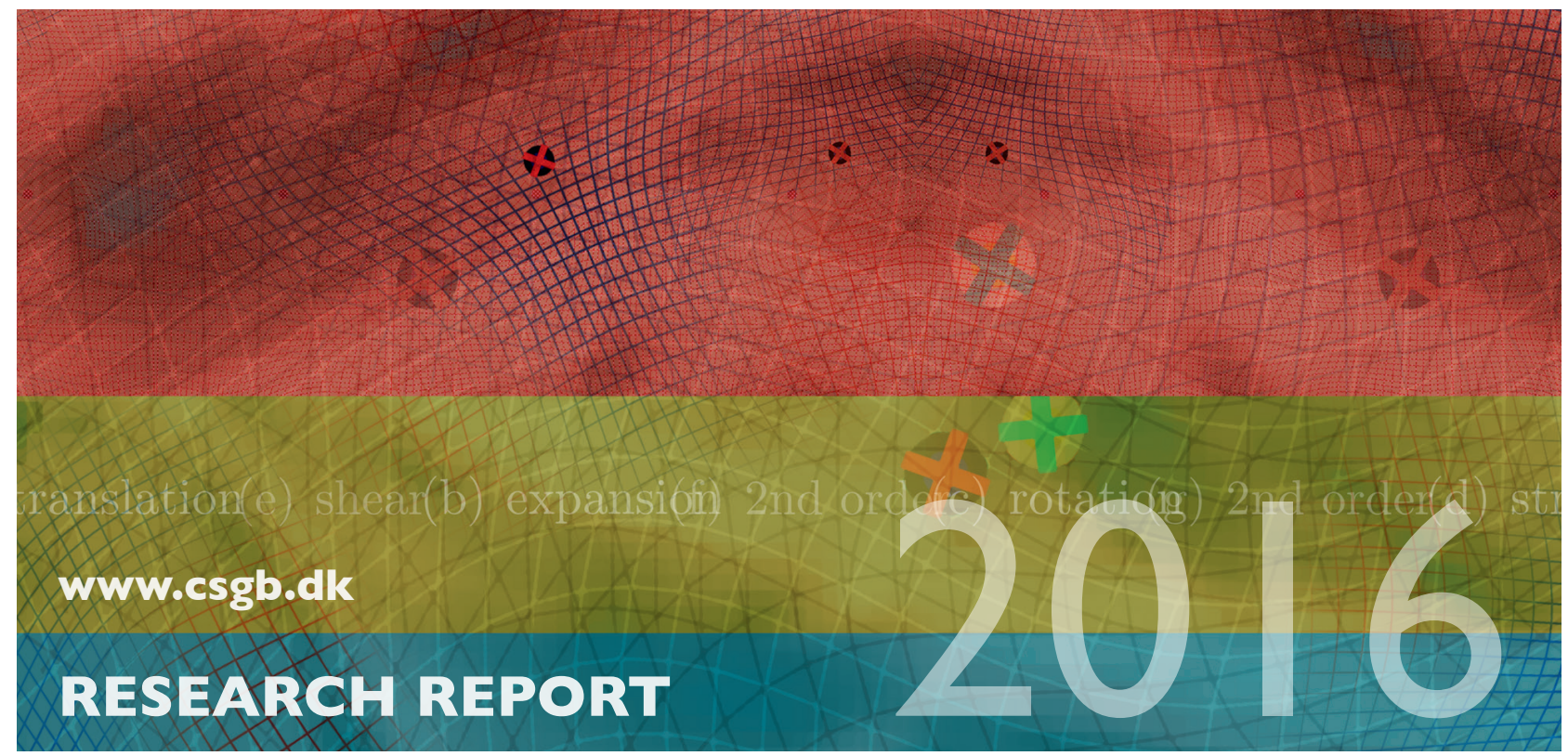

Eva B.Vedel Jensen and Markus Kiderlen

Rotation Invariant Valuations

No. 03, February 2016 


\title{
Rotation Invariant Valuations
}

\author{
Eva B.Vedel Jensen and Markus Kiderlen \\ Department of Mathematics \\ Aarhus University \\ eva@math.au.dk, kiderlen@math.au.dk
}

\begin{abstract}
This paper contains an overview of the results available in the literature, concerning characterization of rotation invariant valuations. In particular, we discuss the characterization theorem, derived in [1], for continuous rotation invariant polynomial valuations on the set $\mathcal{K}^{n}$ of convex bodies in $\mathbb{R}^{n}$. Next, rotational Crofton formulae are presented. Using new kinematic formulae for trace-free tensor valuations, it is possible to extend the rotational Crofton formulae for tensor valuations, available in the literature. Principal rotational formulae for tensor valuations are also discussed. These formulae can be derived using locally defined tensor valuations. A number of open questions in rotational integral geometry are presented.
\end{abstract}

\section{Preliminaries}

The Grassmannian of $q$-dimensional linear subspaces of $\mathbb{R}^{n}$ is denoted by $G(n, q)$, $0 \leq q \leq n$. For $L \in G(n, q)$, the set $G(L, p)$ is the family of all $p$-dimensional linear subspaces $M$ incident with $L$, that is, $M \subset L$ when $p \leq q$ and $L \subset M$, otherwise. The invariant probability measures on these spaces are denoted by $\nu_{q}$ and $\nu_{p}^{L}$, respectively. Similarly the space $A(n, q)$ of $q$-dimensional flats is endowed with the motion invariant measure $\mu_{q}$, normalized in such a way that

$$
\mu_{q}\left(\left\{E \in A(n, q): E \cap B^{n} \neq \emptyset\right\}\right)=\kappa_{n-q},
$$

where $\kappa_{j}$ is the volume of the Euclidean unit ball $B^{j}$ in $\mathbb{R}^{j}$. For $E \in G(n, q)$ the family of all $p$-dimensional flats incident with $E$ is denoted by $A(E, p)$ and endowed with the invariant measure $\mu_{p}^{E}$. When $q \geq p$ the measure $\mu_{p}^{E}$ is obtained by identifying $E$ with $\mathbb{R}^{q}$ and taking the image measure of $\mu_{p}$ in $\mathbb{R}^{q}$ using this identification. When $q<p$ and $E=L+x$ with $x \in L^{\perp}$, the measure $\mu_{p}^{E}$ is the image measure of $\mu_{n-q}^{L^{\perp}}$ under the mapping $N \mapsto N^{\perp}+x$.

The subspace determinant $[L, M]$ of two flats $L$ and $M$ is defined in [28, Section 14.1]. Let $\mathcal{K}^{n}$ be the family of convex bodies, that is, of all non-empty compact convex subsets of $\mathbb{R}^{n}$. For $E \in A(n, q)$ we let $\mathcal{K}_{E}^{q}$ be the family of all convex bodies in $E$. The unit normal bundle of a set of positive reach $X$ is nc $X$. 
We will need a norm on the space $\mathbb{T}^{p}$ of symmetric tensors of rank $p \in \mathbb{N}_{0}$ and define

$$
\|T\|=\sup \left\{\left|T\left(v_{1}, \ldots, v_{p}\right)\right|:\left\|v_{1}\right\|, \ldots,\left\|v_{p}\right\| \leq 1\right\}
$$

for $T \in \mathbb{T}^{p}$.

We will make use of Gauss' hypergeometric function

$$
F_{\alpha, \beta ; \gamma}(z)=\sum_{k=0}^{\infty} \frac{(\alpha)_{k}(\beta)_{k}}{(\gamma)_{k}} \frac{z^{k}}{k !}
$$

$\alpha, \beta, \gamma \in \mathbb{R},-\gamma \notin \mathbb{N}_{0}$, where $(\alpha)_{k}=\alpha(\alpha+1) \cdots(\alpha+k-1)$. The series in (1.1) converges absolutely for $z \in(-1,1)$ and if $\alpha+\beta<\gamma$ even for $z \in[-1,1]$. We will later use one of Euler's transformation rules

$$
F_{\alpha, \beta ; \gamma}(z)=(1-z)^{\gamma-(\alpha+\beta)} F_{\gamma-\alpha, \gamma-\beta ; \gamma}(z)
$$

and the fact that

$$
F_{\alpha, \alpha+\frac{1}{2} ; 2 \alpha}(z)=(1-z)^{-1 / 2}\left(\frac{1+\sqrt{1-z}}{2}\right)^{1-2 \alpha},
$$

$|z|<1$; see, for instance, [7, (8.2.11) and p. 296].

\section{Rotation invariant continuous valuations on star sets}

Before describing rotation invariant valuations on the family of convex bodies, we describe here shortly a theory of rotation invariant tensor valuations for star sets. With the appropriate definition of star sets, this theory turns out to be rather complete and can serve as a reference for the convex case that still contains a number of open questions.

A set $S \subset \mathbb{R}^{n}$ is called star shaped if its intersection with an arbitrary line through the origin $o$ is a (possibly degenerate) line-segment. Clearly, a star shaped set $S$ is determined by its radial function

$$
\rho(S, u)=\sup \{\alpha \in \mathbb{R}: \alpha u \in S\},
$$

$u \in \mathbb{S}^{n-1}$. Usually one only works with geometrically defined subclasses of the family of all star shaped sets, and results depend crucially on the subclass chosen. In this survey we restrict considerations exclusively to star shaped sets containing the origin. Note that the results on star bodies in Gardner's monograph [13] do not require this assumption. We base our review on Klain's [22] definition of an $L^{n}$-star, which is a star shaped set $S \subset \mathbb{R}^{n}$ that contains the origin and has a finite volume, that is, its radial function is a non-negative element of $L^{n}\left(\mathcal{H}^{n-1}\right)$. The family of all $L^{n}$-stars will be denoted by $\mathcal{S}^{n}$, and endowed with the topology that is induced by the norm in $L^{n}\left(\mathcal{H}^{n-1}\right)$ on $\left\{\rho(S, \cdot): S \in \mathcal{S}^{n}\right\}$. As usual, one thus identifies $L^{n}$-stars when their radial functions coincide up to a set of $\mathcal{H}^{n-1}$-measure zero. 
We now discuss examples of continuous $\mathrm{SO}(n)$-invariant valuations on $\mathcal{S}^{n}$ that take values in the space of tensors of rank $p \in \mathbb{N}_{0}$. The first examples that come to mind are the Euler-Poincaré characteristic

$$
\chi(S)=1,
$$

and, of course, the volume

$$
\lambda_{n}(S)=\int_{S} 1 \mathrm{~d} x
$$

$S \in \mathcal{S}^{n}$, yielding tensor valued valuations of rank 0. To obtain higher rank tensors, the constant 1 in (2.1) and in the integrand of (2.2) can be replaced by tensors - in the second case possibly one that depends on $x$. To retain the rotation invariance, this tensor must depend on $x$ only through $\|x\|$, so we may put

$$
\varphi(S)=T+\int_{S} f(\|x\|) \mathrm{d} x
$$

with some fixed $T \in \mathbb{T}^{p}$ and a suitable function $f:[0, \infty) \rightarrow \mathbb{T}^{p}$. Rewriting (2.3) using polar coordinates gives

$$
\varphi(S)=\int_{\mathbb{S}^{n-1}} \theta(\rho(S, u)) \mathrm{d} u
$$

where $\theta:[0, \infty) \rightarrow \mathbb{T}^{p}$ must be continuous, as the restriction of $\varphi$ on $\left\{\alpha B^{n}: \alpha \geq 0\right\}$ is continuous. Finally, to assure that (2.4) defines a tensor valued mapping on $\mathcal{S}^{n}, \theta(t)$ may not grow faster than $t^{n}$ as $t \rightarrow \infty$; see [23, Lemma 2.2]. With these conditions on $\theta$, we have found all rotation invariant continuous valuations on $\mathcal{S}^{n}$.

Theorem 1 (Klain [23, Theorem 2.8]). For every $\mathrm{SO}(n)$-invariant continuous valuation $\varphi: \mathcal{S}^{n} \rightarrow \mathbb{T}^{p}$ there is a continuous function $\theta:[0, \infty) \rightarrow \mathbb{T}^{p}$ with $\|\theta(t)\| \leq a t^{n}+b$, $t \geq 0$, for some $a, b \geq 0$ such that (2.4) holds.

Conversely, for any $\theta$ as above, (2.4) defines an $\mathrm{SO}(n)$-invariant continuous valuation on $\mathcal{S}^{n}$ with values in $\mathbb{T}^{p}$.

Klain stated this result only for $p=0$ but it can easily be extended to positive $p$ by pointwise application to the tensors involved. The proof of Theorem 1 relies on the fact that the family $\mathcal{S}^{n}$ is very large. To illustrate the main idea restrict considerations to the case where $p=0$. For any $r>0$ the functional $\mu$ given by

$$
\mu(A)=\varphi(\{t a: 0 \leq t \leq r, a \in A\}),
$$

for measurable $A \subset \mathbb{S}^{n-1}$, is finitely additive. Continuity and the valuation property of $\varphi$ imply that $\mu$ is $\sigma$-additive, and hence $\mu$ is a (possibly signed) measure on $\mathbb{S}^{n-1}$. The measure $\mu$ inherits the rotation invariance from $\varphi$, so $\mu$ must be a multiple $\theta(r)$ of the uniform measure on $\mathbb{S}^{n-1}$. The proof is concluded by observing that any element of $\mathcal{S}^{n}$ can be approximated by finite unions of bounded cones with different $r$, as defined on the right hand side of (2.5). Concluding, the theory of invariant measures is the backbone of Theorem 1. 
Consider an $\mathrm{SO}(n)$-invariant continuous valuation $\varphi: \mathcal{S}^{n} \rightarrow \mathbb{T}^{p}$ with $p=0$. If $\varphi$ is homogeneous of degree $\alpha \in \mathbb{R}$, Theorem 1 implies that $0 \leq \alpha \leq n$. If $\alpha=i$ is an integer, the associated function $\theta$ in (2.4) must be proportional to $t^{i}$, and hence

$$
\varphi=\frac{\varphi\left(B^{n}\right)}{\kappa_{n}} \tilde{W}_{n-i}
$$

is proportional to the $(n-i)$ th dual quermassintegral

$$
\tilde{W}_{n-i}(S)=\frac{1}{n} \int_{\mathbb{S}^{n-1}} \rho(S, u)^{i} \mathrm{~d} u
$$

$S \in \mathcal{S}^{n}$

Of course, Theorem 1 also applies to valuations $\varphi_{L}$ on the subfamily $\mathcal{S}_{L}^{q}$ of all $L^{q}$-stars in a fixed subspace $L \in G(n, q)$, when we identify $L$ with $\mathbb{R}^{q}$, where $q \in$ $\{1, \ldots, n-1\}$. Hence, if $\varphi_{L}: \mathcal{S}_{L}^{q} \rightarrow \mathbb{T}^{p}$ is a continuous valuation that is $\operatorname{SO}(q)$ invariant (with respect to all rotations leaving $L$ fixed), we have

$$
\varphi_{L}(S)=\int_{S^{n-1} \cap L} \theta_{L}(\rho(S, u)) \mathrm{d} u,
$$

$S \in \mathcal{S}_{L}^{q}$. This opens the door to applications in rotational integral geometry. In many practically relevant cases, $\varphi_{L}$ are given for all $L \in G(n, q)$, and are compatible with rotations in the following sense:

$$
\varphi_{L}(S)=\varphi_{\vartheta L}(\vartheta S)
$$

for all $\vartheta \in \operatorname{SO}(n), L \in G(n, q)$ and sets $S \in \mathcal{S}_{L}^{q}$. A family $\left\{\varphi_{L}: L \in G(n, q)\right\}$ of mappings $\varphi_{L}: \mathcal{S}_{L}^{q} \rightarrow \mathbb{T}^{p}$ that satisfies $(2.7)$ is called $\mathrm{SO}(n)$-compatible. Note that in this case all $\varphi_{L}$ are $\mathrm{O}(n)$-invariant on $\mathcal{S}_{L}^{q}$, and if all $\varphi_{L}$ are continuous valuations on $\mathcal{S}_{L}^{q}$, their associated functions $\theta_{L}$ in (2.6) all coincide. In the following we use that when $S \in \mathcal{S}^{n}$, then $S \cap L \in \mathcal{S}_{L}^{q}$ for almost all $L \in G(n, q)$.

Corollary 2. Let $q \in\{1, \ldots, n-1\}$ and let $\left\{\varphi_{L}: L \in G(n, q)\right\}$ be a $\operatorname{SO}(n)$ compatible family of continuous valuations with values in $\mathbb{T}^{p}$. Let $\theta=\theta_{L}$ be the joint associated function in (2.6). Then

$$
\int_{G(n, q)} \varphi_{L}(S \cap L) \nu_{q}(\mathrm{~d} L)=\frac{\omega_{q}}{\omega_{n}} \int_{\mathbb{S}^{n-1}} \theta(\rho(S, u)) \mathrm{d} u
$$

$S \in \mathcal{S}^{n}$

Example 3. For fixed $q \in\{1, \ldots, n-1\}$ and $i \in\{0, \ldots, q\}$ the family $\left\{\tilde{W}_{q-i, L}\right.$ : $L \in G(n, q)\}$ of $(q-i)$ th dual quermass integrals

$$
\tilde{W}_{q-i, L}=\frac{1}{q} \int_{\mathbb{S}^{n-1} \cap L} \rho(\cdot, u)^{i} \mathrm{~d} u,
$$

is $\mathrm{SO}(n)$-compatible. Equation (2.8) now reads

$$
\int_{G(n, q)} \tilde{W}_{q-i, L}(S \cap L) \nu_{q}(\mathrm{~d} L)=\frac{\kappa_{q}}{\kappa_{n}} \tilde{W}_{n-i}(S)
$$

$S \in \mathcal{S}^{n}$. This is the dual Kubota integral recursion essentially due to Lutwak [25]. 
Corollary 4. Let $q \in\{1, \ldots, n-1\}$ and assume that $\varphi: \mathcal{S}^{n} \rightarrow \mathbb{T}^{p}$ is an $\mathrm{SO}(n)$ invariant continuous valuation on $\mathcal{S}^{n}$ such that the associated function in (2.4) satisfies $\|\theta(t)\| \leq a t^{q}+b$ for some $a, b \geq 0$. Then $\varphi$ can be written as a rotational Crofton integral with q-planes:

$$
\int_{G(n, q)} \varphi_{L}(S \cap L) \nu_{q}(\mathrm{~d} L)=\varphi(S)
$$

$S \in \mathcal{S}^{n}$, where

$$
\varphi_{L}\left(S^{\prime}\right)=\frac{\omega_{n}}{\omega_{q}} \int_{\mathbb{S}^{n-1} \cap L} \theta\left(\rho\left(S^{\prime}, u\right)\right) \mathrm{d} u
$$

$S^{\prime} \in \mathcal{S}_{L}^{q}$

Note that if $\left\{\varphi_{L}: L \in G(n, q)\right\}$ is an $\mathrm{SO}(n)$-compatible family of continuous valuations satisfying (2.9), it must be given by (2.10), as such valuations are determined by their values on balls.

It should be mentioned that there are other, more geometrically motivated notions of star sets in the literature. One common class is defined as the family of all star shaped sets containing the origin and having a continuous radial function. Its elements are called star bodies in [32]. Let $\mathcal{S}$ be the family of all star bodies, endowed with the $L^{\infty}$-topology, which is induced by the supremum norm of the radial functions. As $\mathcal{S} \subsetneq \mathcal{S}^{n}$ the above results do not readily apply to valuations on $\mathcal{S}$. However, Villanueva [32] showed that a real-valued $\mathrm{SO}(n)$-invariant $L^{\infty}$-continuous valuation $\varphi$ that is in addition non-negative and satisfies $\varphi(\{o\})=0$, can be written in the form (2.4) with a non-negative continuous function $\theta$ satisfying $\theta(0)=0$. The converse being obvious, this gives a strengthened version of Theorem 1 for star bodies and $p=0$, but only for non-negative valuations with $\varphi(\{o\})=0$. If the latter two conditions are necessary is an open question.

Example 5. The associated function of the real-valued continuous $\mathrm{SO}(n)$-invariant valuation $\varphi(S)=\lambda_{n}(S)$ is $\theta(t)=t^{n} / n$. For $q<n$ there cannot be an $\mathrm{SO}(n)$ compatible family $\left\{\varphi_{L}: L \in G(n, q)\right\}$ of continuous valuations satisfying (2.9), as the joint associated function $\theta$ would be $\left(\omega_{n} /\left(n \omega_{q}\right)\right) t^{n}$, which increases faster than $t^{q}$ as $t \rightarrow \infty$. We thus consider $\varphi$ only on the class of star bodies.

With the same arguments that led to Corollary 4 , we have for any $q \in\{1, \ldots$, $n-1\}$ that

$$
\int_{G(n, q)} \varphi_{L}(S \cap L) \nu_{q}(\mathrm{~d} L)=\lambda_{n}(S)
$$

for any star body $S$, where

$$
\varphi_{L}(S \cap L)=\frac{\omega_{n}}{n \omega_{q}} \int_{\mathbb{S}^{n-1} \cap L} \rho(S, u)^{n} \mathrm{~d} u=\frac{\omega_{n}}{\omega_{q}} \int_{S \cap L}\|x\|^{n-q} \mathrm{~d} x .
$$

This is a special case of the rotational Crofton formula for intrinsic volumes in [5]. 


\section{Rotation invariant continuous valuations on convex bodies}

We now turn to rotation invariant continuous valuations on the family of convex bodies, endowed with the Hausdorff metric. Throughout the rest of this paper we assume $n \geq 2$ to avoid peculiarities of the one-dimensional setting.

Clearly, valuations of the form (2.3), restricted to $\mathcal{K}^{n}$, are examples of continuous $\mathrm{SO}(n)$-invariant valuations, but the family of continuous $\mathrm{SO}(n)$-invariant valuations on $\mathcal{K}^{n}$ is much richer. One simple example are the intrinsic volumes $V_{j}, 0<j<n$, they are even motion invariant, but not of the form (2.3).

In the seminal paper [1] by Alesker, characterization theorems for rotation invariant continuous polynomial valuations are derived. A valuation $\varphi: \mathcal{K}^{n} \rightarrow \mathbb{T}^{p}$ is called polynomial of degree at most $k$ if $\varphi(K+x)$ is a polynomial in $x$ of degree at most $k$ for all $K \in \mathcal{K}^{n}$. If $\varphi$ is polynomial of degree at most $k$ and $\varphi(K+x)$ is a polynomial in $x$ of exact degree $k$ for at least one $K \in \mathcal{K}^{n}, \varphi$ is called polynomial of degree $k$.

In [1], a characterization theorem for continuous polynomial rotation invariant valuations is derived, involving the family of valuations given by

$$
\varphi_{p, j}(K)=\int_{\mathbf{n c} K} p\left(\|x\|^{2},\langle x, u\rangle\right) \Lambda_{j}(K, \mathrm{~d}(x, u))
$$

where $p$ is a polynomial in two variables with values in $\mathbb{T}^{p}$ and $j \in\{0, \ldots, n-1\}$. The properties of the support measures $\Lambda_{j}(K, \cdot)$, listed for instance in [27, Section 4.2], imply that $\varphi_{p, j}: \mathcal{K}^{n} \rightarrow \mathbb{T}^{p}$ is an $\mathrm{O}(n)$-invariant continuous valuation. In addition, $\varphi_{p, j}$ is a polynomial valuation of degree at $\operatorname{most} 2 \operatorname{deg} p$.

Theorem 6 (Alesker [1, Theorem B (i)]). For every continuous polynomial valuation $\varphi: \mathcal{K}^{n} \rightarrow \mathbb{T}^{p}$, which is $\mathrm{SO}(n)$-invariant if $n \geq 3$ and $\mathrm{O}(n)$-invariant if $n=2$, there exist polynomials $p_{0}, \ldots, p_{n-1}$ in two variables with values in $\mathbb{T}^{p}$ such that

$$
\varphi=\sum_{j=0}^{n-1} \varphi_{p_{j}, j} .
$$

Conversely, any expression of the form (3.2) defines a continuous polynomial $\mathrm{O}(n)$ invariant valuation on $\mathcal{K}^{n}$ with values in $\mathbb{T}^{p}$.

Note that as (3.1) defines an $\mathrm{O}(n)$-invariant valuation, every continuous polynomial $\mathrm{SO}(n)$-invariant valuation is also $\mathrm{O}(n)$-invariant when $n \geq 3$. A characterization theorem for the particular case of continuous polynomial $\mathrm{SO}(2)$-invariant valuations on $\mathcal{K}^{2}$ can also be found in [1]. As we do not require that the valuations are translation invariant, McMullen's decomposition is not readily available. However, polynomiality of degree at most $k$ implies that $\varphi$ can be decomposed into a sum of homogeneous valuations with homogeneity degrees in $\{0,1, \ldots, n+k\}$. This follows from a more general result in [20] and is used extensively in [1]. 
Example 7. A very simple example of a continuous polynomial $\mathrm{O}(n)$-invariant valuation on $\mathcal{K}^{n}$ is

$$
\varphi_{n}^{k}(K)=\int_{K}\|x\|^{2 k} \mathrm{~d} x
$$

$k \in \mathbb{N}_{0}$. This valuation is of the form (3.2), since the divergence theorem implies

$$
(n+2 k) \int_{K}\|x\|^{2 k} \mathrm{~d} x=2 \int_{\mathbf{n c} K}\|x\|^{2 k}\langle x, u\rangle \Lambda_{n-1}(K, \mathrm{~d}(x, u)),
$$

see e.g. [27, p. 316].

The space $V_{n, k}$ of all real-valued continuous $\mathrm{O}(n)$-invariant (or, equivalently, $\mathrm{SO}(n)$-invariant when $n \geq 3)$ valuations in $\mathbb{R}^{n}$ that are polynomial of degree at most $k \in \mathbb{N}_{0}$ is finite dimensional.

In fact, Alesker has shown the following decomposition

$$
V_{n, k}=W_{n, 0} \oplus W_{n, 1} \oplus \ldots \oplus W_{n, k},
$$

where each subspace $W_{n, q}$ is spanned by valuations of (exact) polynomial degree $q$. This yields the recursive formula

$$
\operatorname{dim} V_{n, k}=\operatorname{dim} V_{n, k-1}+\operatorname{dim} W_{n, k} .
$$

As $\operatorname{dim} W_{n, 2 q+1}=q(n-1)$ and $\operatorname{dim} W_{n, 2 q}=q(n-1)+(n+1)$, see [1, p. 997], this implies

$$
\begin{aligned}
\operatorname{dim} V_{n, 2 i} & =i^{2}(n-1)+(i+1)(n+1), \\
\operatorname{dim} V_{n, 2 i+1} & =i(i+1)(n-1)+(i+1)(n+1),
\end{aligned}
$$

for all $i \in \mathbb{N}_{0}, n \geq 3$. The fact that $\operatorname{dim} V_{n, 0}=\operatorname{dim} W_{n, 0}=n+1$ is a direct consequence of Hadwiger's theorem, as valuations of polynomial degree zero are translation invariant, and thus $V_{0}, \ldots, V_{n}$ forms a basis of $W_{n, 0}$. Furthermore, we see $\operatorname{dim} V_{n, 1}=\operatorname{dim} V_{n, 0}=n+1$, so $W_{n, 1}$ is trivial - continuous $\operatorname{SO}(n)$-invariant valuations of polynomial degree exactly one do not exist. Explicit bases for $W_{n, k}$ and hence for $V_{n, k}$ can be constructed from the family of valuations

$$
\varphi_{j}^{r, s}(K)=\int_{\mathbf{n c} K}\|x\|^{2 r}\langle x, u\rangle^{s} \Lambda_{j}(K, \mathrm{~d}(x, u)),
$$

$r, s \in \mathbb{N}_{0}, j=0, \ldots, n-1$. For odd polynomial degree $k=2 q+1, q \in \mathbb{N}$, the valuations $\varphi_{j}^{q-i, 2 i+1}, j=1, \ldots, n-1, i=1, \ldots, q$, form a basis of $W_{n, 2 q+1}$. For even polynomial degree $k=2 q, q \in \mathbb{N}_{0}$, the valuations $\varphi_{j}^{q-i, 2 i}, j=1, \ldots, n-1$, $i=0, \ldots, q$, (note that $i=0$ is included now) together with $\varphi_{n}^{q}$ and $\varphi_{0}^{q, 0}$ form a basis of $W_{n, 2 q}$. (For the definition of $\varphi_{n}^{q}$, see Example 7.) This follows from the facts that the exact polynomial degree of any of these valuations is $2 q+1$ and $2 q$, respectively, and that these valuations can replace the less explicit ones in [1, Lemma 4.8]. More explicitly for the planar case, a basis of all $\mathrm{O}(2)$-invariant continuous valuations of degree at most $k \in 2 \mathbb{N}_{0}+1$ is given by the valuations $\varphi_{1}^{i, j}$, where the non-negative integers $i$ and $j$ satisfy $2 i+j \leq k$, together with $\varphi_{0}^{2 i, 0}, 0 \leq 2 i<k$.

From the above it is straightforward to find a basis of the space $V_{n, k}^{p}$ of all $\mathbb{T}^{p}$ valued continuous $\mathrm{O}(n)$-invariant (or, equivalently, $\mathrm{SO}(n)$-invariant when $n \geq 3$ ) 
valuations in $\mathbb{R}^{n}$, as any $\varphi \in V_{n, k}^{p}$ can be written as a linear combination of basis vectors in $\mathbb{T}^{p}$, where the coefficients are in $V_{n, k}$. We only note here that this implies

$$
\operatorname{dim} V_{n, k}^{p}=\operatorname{dim} V_{n, k} \cdot \operatorname{dim} \mathbb{T}^{p},
$$

where $\operatorname{dim} \mathbb{T}^{p}=\left(\begin{array}{c}n+p-1 \\ p\end{array}\right)$.

The valuations in (3.1) are all quasi-smooth. A continuous valuation $\varphi: \mathcal{K}^{n} \rightarrow \mathbb{R}$ is called quasi-smooth, if the map on $\mathcal{K}^{n}$ given by

$$
K \mapsto[(t, x) \mapsto \varphi(t K+x)]
$$

$t \in[0,1], x \in \mathbb{R}^{n}$, is a continuous map from $\mathcal{K}^{n}$ into the space $C^{n}\left([0,1] \times \mathbb{R}^{n}\right)$ of $n$-times continously differentiable functions on $[0,1] \times \mathbb{R}^{n}$. This notion is extended to $\mathbb{T}^{p}$-valued valuations by assuming quasi-smoothness pointwise i.e. for all real-valued valuations $\varphi(K)\left(x_{1}, \ldots, x_{p}\right), x_{1}, \ldots, x_{p} \in \mathbb{R}^{n}$.

Alesker [2,3] showed that any quasi-smooth valuation can be approximated uniformly on any compact subset of $\mathcal{K}^{n}$ by continuous polynomial valuations. For the understanding of $\mathrm{SO}(n)$-invariant quasi-smooth valuations it is thus sufficient to investigate the valuations $\varphi_{p, j}$, defined in (3.1). There are $\mathrm{SO}(n)$-invariant continuous valuations that are not quasi-smooth, but it is an open problem if all of them can be approximated by continuous polynomial valuations.

Example 8. On $\mathcal{K}^{2}$ the functional

$$
\varphi(K)=\int_{K}\|x\|^{-1} \mathrm{~d} x
$$

is a real-valued $\mathrm{O}(2)$-invariant continuous valuation (the finiteness of which can be seen by introducing polar coordinates). The valuation $\varphi$ is a special case of the valuations appearing in Theorem 11 below. The valuation is not quasi-smooth. In fact, for $K=[0,1]^{2}$ and $s>0$ an application of the divergence theorem like in Example 7 shows that

$$
\varphi(K+(s, s))=-2 s \int_{s}^{1+s}\|(s, y)\|^{-1} \mathrm{~d} y+2(1+s) \int_{s}^{1+s}\|(1+s, y)\|^{-1} \mathrm{~d} y .
$$

The second derivative of this function of $s$ has a pole at 0 , so $\varphi$ is not quasi-smooth. However, it can be shown that $\varphi$ can be approximated uniformly on any compact subset of $\mathcal{K}^{2}$ by continuous polynomial valuations.

Rotational integral geometry for the valuations appearing in the characterization theorems in [1] appears largely unexplored. Below we show, as a new result, how the valuation $\varphi_{n-1}^{r, s}$ defined in (3.3) with $s$ even can be expressed as a rotational average. The assumption that $s$ is even can be omitted when $o \in K$.

Theorem 9. Let $q \in\{2, \ldots, n-1\}, r, s$ non-negative integers with s even. Then, the valuation $\varphi_{n-1}^{r, s}$ in (3.3) can be written as a rotational Crofton integral with q-planes:

$$
\int_{G(n, q)} \varphi_{L}^{r, s}(K \cap L) \nu_{q}(\mathrm{~d} L)=\varphi_{n-1}^{r, s}(K)
$$


for all $K \in \mathcal{K}^{n}$. Here

$$
\varphi_{L}^{r, s}\left(K^{\prime}\right)=\frac{\omega_{n}}{\omega_{q}} \int_{\mathbf{n c}\left(K^{\prime}\right)}\|x\|^{2 r+n-q}\langle x, u\rangle^{s} F_{\frac{s-1}{2},-\frac{n-q}{2} ; \frac{q-1}{2}}\left(\sin ^{2} \angle(x, u)\right) \Lambda_{q-1}^{L}\left(K^{\prime}, \mathrm{d}(x, u)\right)
$$

is an integral with respect to the generalized curvature measure $\Lambda_{q-1}^{L}\left(K^{\prime}, \cdot\right)$ of $K^{\prime} \in$ $\mathcal{K}_{L}^{q}$ relative to $L$.

Proof. As support measures are weakly continuous and the integrand in the definition of $\varphi_{L}^{r, s}$ is continuous in $(x, u)$, one can apply an approximation argument. It is thus enough to show the claim for a polytope $K$ for which the union of all support planes of $K$ at the facets does not contain the origin. The variable $s$ is even, so it does not matter if one works with the exterior or the interior normal vectors. It is thus enough to show the claim for one facet, or, equivalently, for all $(n-1)$-dimensional sets $K$. Let $u \in \mathbb{S}^{n-1}$ be one of the unit normals of $K$ at a relative interior point. Then

$$
\varphi_{n-1}^{r, s}(K)=\int_{K}\|x\|^{2 r}\langle x, u\rangle^{s} \mathcal{H}^{n-1}(\mathrm{~d} x)
$$

and using [16, Proposition 5.4] we find

$$
\varphi_{r, s}(K)=\frac{\omega_{n}}{2} \int_{G(n, 1)} \int_{K \cap M}\|x\|^{2 r+s+n-1}\left[u^{\perp}, M\right]^{s-1} \mathcal{H}^{0}(\mathrm{~d} x) \nu_{1}(\mathrm{~d} M) .
$$

The only analytic function $h$ that satisfies

$$
\int_{G(M, q)} h\left(\left[u^{\perp} \cap L, M\right]^{s-1}\right) \nu_{q}^{M}(\mathrm{~d} L)=\left[u^{\perp}, M\right]^{s-1}
$$

for all $M \in G(n, 1)$ is given by

$$
h(z)=z F_{\frac{s-1}{2},-\frac{n-q}{2} ; \frac{q-1}{2}}\left(1-z^{\frac{2}{s-1}}\right) .
$$

The proof of this claim follows closely [16, Section 5.6], where the case $s=0$ is treated. Using (3.5) and interchanging the order of integration we find

$$
\varphi_{r, s}(K)=\int_{G(n, q)} \varphi_{L}^{r, s}(K \cap L) \nu_{q}(\mathrm{~d} L)
$$

with

$$
\begin{aligned}
\varphi_{L}^{r, s}(K \cap L) & =\frac{\omega_{n}}{2} \int_{G(L, 1)} \int_{(K \cap L) \cap M}\|x\|^{2 r+s+n-1} h\left(\left[u^{\perp} \cap L, M\right]^{s-1}\right) \mathcal{H}^{0}(\mathrm{~d} x) \nu_{1}^{L}(\mathrm{~d} M) \\
& =\frac{\omega_{n}}{\omega_{q}} \int_{K \cap L}\|x\|^{2 r+s+n-q}\left[u^{\perp} \cap L, M_{x}\right] h\left(\left[u^{\perp} \cap L, M_{x}\right]^{s-1}\right) \mathcal{H}^{q-1}(\mathrm{~d} x),
\end{aligned}
$$

where we at the last equality sign have again used [16, Proposition 5.4], but now in $L$, and we wrote $M_{x}$ for $\operatorname{span}\{x\}$. As $\left[u^{\perp} \cap L, M_{x}\right]$ is the cosine of the angle between $x$ and the unit normal vector of $K \cap L$ in $L$, this function $\varphi_{L}^{r, s}$ coincides with the one in the statement of the theorem. 
Rotational integral geometry of intrinsic volumes has been developed during the last decade in a series of papers $([4,5,10,18])$, motivated by the strong interest in such results from local stereology ([16]). In the theorem below, we show in the spirit of Corollary 4 how the intrinsic volumes can be expressed as rotational averages. A central element in the proof of the theorem is the classical Crofton formula for affine subspaces

$$
\int_{A(n, q)} V_{j}(K \cap E) \mu_{q}(\mathrm{~d} E)=\alpha_{n, j, q} V_{n+j-q}(K),
$$

where

$$
\alpha_{n, j, q}=\frac{\left(\begin{array}{c}
q \\
j
\end{array}\right) \kappa_{q} \kappa_{n+j-q}}{\left(\begin{array}{c}
n \\
q-j
\end{array}\right) \kappa_{j} \kappa_{n}},
$$

and $0 \leq j \leq q \leq n$; see [27, Section 4.4].

Theorem 10 (Auneau \& Jensen [5], Gual-Arnau et al. [10]). For $q=1, \ldots, n-1$ and $j=1, \ldots, q$, let $\varphi=V_{n+j-q}$ be the intrinsic volume of homogeneity degree $n+j-q$. Then,

$$
\int_{G(n, q)} \varphi_{L}(K \cap L) \nu_{q}(\mathrm{~d} L)=\varphi(K)
$$

$K \in \mathcal{K}^{n}$, where

$$
\varphi_{L}\left(K^{\prime}\right)=\frac{\omega_{n-q+1}}{\omega_{1}} \frac{1}{\alpha_{n, j-1, q-1}} \int_{A(L, q-1)} d(o, E)^{n-q} V_{j-1}\left(K^{\prime} \cap E\right) \mu_{q-1}(\mathrm{~d} E),
$$

$K^{\prime} \in \mathcal{K}_{L}^{q}$, and $d(o, E)$ is the distance from o to $E$. For $j=q$, (3.8) takes the following explicit form

$$
\varphi_{L}\left(K^{\prime}\right)=\frac{\omega_{n}}{\omega_{q}} \int_{K^{\prime}}\|x\|^{n-q} \mathrm{~d} x
$$

while for $j=q-1$, (3.8) can equivalently be expressed as

$$
\varphi_{L}\left(K^{\prime}\right)=\frac{\omega_{n}}{\omega_{q}} \int_{\mathbf{n c}\left(K^{\prime}\right)}\|x\|^{n-q} F_{-\frac{1}{2},-\frac{n-q}{2} ; \frac{q-1}{2}}\left(\sin ^{2} \angle(x, u)\right) \Lambda_{q-1}^{L}\left(K^{\prime}, \mathrm{d}(x, u)\right) .
$$

Note that (3.9) also appears in Example 5, while (3.10) is obtained by setting $r=s=0$ in Theorem 9 and noting that $\varphi_{n-1}^{0,0}(K)=2 V_{n-1}(K)$.

Besides the classical Crofton formula, the proof of Theorem 10 uses the following version of the Blaschke-Petkantschin formula for a non-negative measurable function $f$ on $A(n, r)$, see [21, Theorem 2.7],

$$
\int_{A(n, r)} f(E) \mu_{r}(\mathrm{~d} E)=\frac{\omega_{n-r}}{\omega_{q-r}} \int_{G(n, q)} \int_{A(L, r)} d(o, E)^{n-q} f(E) \mu_{r}^{L}(\mathrm{~d} E) \nu_{q}(\mathrm{~d} L),
$$

$q=1, \ldots, n-1, r=0, \ldots, q-1$. This formula, also called the invariator principle in stereology $([31])$, is used to translate the classical Crofton formula, dealing with 
affine subspaces, into a result for linear subspaces. The details of the proof may be found in [17, p. 239].

The formula for $\varphi_{L}$ in (3.8) is not very explicit, but actually useful in local stereology, because a stereological estimator of $V_{n-q+j}(K)$ can be constructed from this formula, involving motion invariant random flats within isotropic random linear subspaces, as explained in Section 7 below. However, from a theoretical point of view, a more explicit expression for (3.8) would be desirable. To the best of our knowledge, this is an open problem in rotational integral geometry.

In the spirit of Corollary 2 , we now consider the $\mathrm{SO}(n)$-compatible family $\left\{\varphi_{L}\right.$ : $L \in G(n, q)\}$ where

$$
\varphi_{L}\left(K^{\prime}\right)=V_{j}\left(K^{\prime}\right),
$$

$K^{\prime} \in \mathcal{K}_{L}^{q}, q=1, \ldots, n-1, j=0, \ldots, q$. In $[4,18]$, the rotational averages of these sectional valuations are derived. The result is presented in the theorem below.

Theorem 11 (Auneau, Rataj \& Jensen $[4,18]$ ). Let $q=1, \ldots, n-1, j=0, \ldots, q$ and $\left\{\varphi_{L}: L \in G(n, q)\right\}$ be the $\mathrm{SO}(n)$-compatible family given by (3.12). Then,

$$
\int_{G(n, q)} \varphi_{L}(K \cap L) \nu_{q}(\mathrm{~d} L)=\varphi(K)
$$

where for $j=q$

$$
\varphi(K)=\frac{\omega_{q}}{\omega_{n}} \int_{K}\|x\|^{-(n-q)} \mathrm{d} x .
$$

If $o \notin \mathrm{bd} K$, then for $j<q$

$$
\begin{aligned}
\varphi(K)= & \frac{2 \omega_{q}}{\omega_{n} \omega_{q-j}} \int_{\mathbf{n c} K}\|x\|^{-(n-q)} \\
& \times \sum_{\substack{I \subset\{1, \ldots, n-1\} \\
|I|=q-j-1}} Q_{q}\left(x, u, A_{I}\right) \frac{\prod_{i \in I} \kappa_{i}(x, u)}{\prod_{i=1}^{n-1} \sqrt{1+\kappa_{i}^{2}(x, u)}} \Lambda_{n-1}(K, \mathrm{~d}(x, u)),
\end{aligned}
$$

where $\kappa_{i}(x, u), i=1, \ldots, n-1$, are the principle curvatures of $\mathbf{n c} K$ at $(x, u)$. Furthermore, $A_{I}=A_{I}(x, u)$ is the $(n-1-|I|)$-dimensional subspace spanned by the principal directions $a_{i}(x, u), i \notin I$, at $(x, u) \in \mathbf{n c} K$, and

$$
Q_{q}\left(x, u, A_{I}\right)=\int_{G(\operatorname{span}\{x\}, q)} \frac{\left[L, A_{I}\right]^{2}}{\left\|p_{L} u\right\|^{q-j}} \nu_{q}^{\operatorname{span}\{x\}}(\mathrm{d} L) .
$$

If $q=1$ and $x \perp u$, we set $Q_{1}(x, u, M)=0$. For $j=q-1$, (3.14) takes the following explicit form

$$
\varphi(K)=\frac{\omega_{q}}{\omega_{n}} \int_{\mathbf{n c} K}\|x\|^{-(n-q)} F_{-\frac{1}{2}, \frac{n-q}{2} ; \frac{n-1}{2}}\left(\sin ^{2} \angle(x, u)\right) \Lambda_{n-1}(K, \mathrm{~d}(x, u)) .
$$

The proof of the theorem involves extensive geometric measure theory.

In [4], the explicit form of $Q_{q}$ has been derived. Generally, $Q_{q}\left(x, u, A_{I}\right)$ depends on the angle between $x$ and $u$, and the angle between $x$ and $A_{I}$. As an example, let 
$j=0$ and $q=n-1$. Then, by [18, Proposition 3],

$$
\begin{aligned}
\varphi(K)= & \frac{2}{(n-1) \omega_{n}} \int_{\mathbf{n c} K}\|x\|^{-1} \\
& \times\left[\sum_{i=1}^{n-1} R\left(x, u, a_{i}(x, u)\right) \frac{\prod_{j \neq i} \kappa_{j}(x, u)}{\prod_{l=1}^{n-1} \sqrt{1+\kappa_{l}^{2}(x, u)}}\right] \Lambda_{n-1}(K, \mathrm{~d}(x, u)),
\end{aligned}
$$

where

$$
\begin{aligned}
R(x, u, a)= & \sin ^{2} \angle(x, a)\left[\sin ^{2} \theta F_{\frac{n-1}{2}, \frac{1}{2} ; \frac{n+1}{2}}\left(\sin ^{2} \angle(x, u)\right)\right. \\
& \left.+\cos ^{2} \theta F_{\frac{n-1}{2}, \frac{3}{2} ; \frac{n+1}{2}}\left(\sin ^{2} \angle(x, u)\right)\right]
\end{aligned}
$$

with $\theta=\angle\left(p_{x^{\perp}} a, p_{x^{\perp}} u\right)$. For $n=3$ an application of the Euler-transformation (1.2) implies

$$
R(x, u, a)=F_{1, \frac{3}{2} ; 2}\left(\sin ^{2} \angle(x, u)\right) \sin ^{2} \angle(x, a)\left[\left(\sin ^{2} \theta\right) \cos \angle(x, u)+\cos ^{2} \theta\right] .
$$

As

$$
\cos \theta=\frac{\cos \angle(a, x) \cos \angle(x, u)}{\sin \angle(a, x) \sin \angle(x, u)},
$$

trigonometric identities give

$$
\begin{aligned}
R(x, u, a)= & F_{1, \frac{3}{2} ; 2}\left(\sin ^{2} \angle(x, u)\right) \\
& \times\left[\left(\sin ^{2} \angle(x, a)\right) \cos \angle(x, u)+2 \cos ^{2} \angle(x, a) \frac{\cos ^{2} \angle(x, u)}{\sin ^{2} \angle(x, u)} \sin ^{2} \frac{\angle(x, u)}{2}\right],
\end{aligned}
$$

where $F_{1, \frac{3}{2} ; 2}$ can be simplified using (1.3) with $\alpha=1$. Summarizing, we find for $n=3, q=2$ and $j=0$ that (3.14) reduces to

$$
\begin{aligned}
\varphi(K)= & \frac{1}{8 \pi} \int_{\mathbf{n c} K}\|x\|^{-1}\left[\sum _ { i = 1 } ^ { 2 } \frac { \kappa _ { 3 - i } ( x , u ) } { \prod _ { l = 1 } ^ { 2 } \sqrt { 1 + \kappa _ { l } ^ { 2 } ( x , u ) } } \left[\sin ^{2} \angle\left(x, a_{i}(x, u)\right) \cos ^{-2} \frac{\angle(x, u)}{2}\right.\right. \\
& \left.\left.+2 \cos ^{2} \angle\left(x, a_{i}(x, u)\right) \frac{\cos \angle(x, u)}{\sin ^{2} \angle(x, u)} \tan ^{2} \frac{\angle(x, u)}{2}\right]\right] \mathcal{H}^{2}(\mathrm{~d}(x, u)) .
\end{aligned}
$$

We conclude these considerations with a remark on $\mathrm{SO}(n)$-invariant valuations in the context of the above rotational formulae. When $\varphi_{L}=V_{j}$ is an intrinsic volume, the left hand side of (3.13) defines a real-valued $\mathrm{SO}(n)$-invariant valuation $\varphi$. In the case of the Euler characteristic, $j=0$, the valuation $\varphi$ is not continuous, as can be seen considering a non-constant sequence of singletons converging to $\{o\}$. Using the upper semi-continuity of the intersection operation one can show that $\varphi$ is continuous for $j \geq 1$. One may ask if this valuation can be approximated by polynomial ones. Due to Weierstrass' approximation theorem the hypergeometric function in (3.15) can uniformly be approximated by polynomials on $[-1,1]$. As a consequence, the valuation in (3.15) is a locally uniform limit of continuous $\mathrm{SO}(n)$-invariant polynomial valuations by Theorem 6 . In contrast to this, the valuation in (3.14) is for $j \leq q-2$ an integral over the unit normal bundle where the integrand depends on the principal directions of nc $K$. It was therefore conjectured in [4] that such valuations are not locally uniform limits of continuous $\mathrm{SO}(n)$-invariant polynomial valuations even if $j \geq 1$. The lowest dimensional example of this kind occurs for $n=4, q=3$ and $j=1$. The mentioned problem is still open. 


\section{Rotational Crofton formulae for Minkowski tensors}

Rotational Crofton formulae for Minkowski tensors have recently been derived in $[6,30]$.

To express Minkowski tensors as rotational averages, we need to generalize Theorem 10. An important element in the proof of Theorem 10 is the classical Crofton formula (3.7). In [15], (3.7) is generalized to the case of Minkowski tensors. It turns out that the formula for Minkowski tensors derived in [15] is considerably more complicated than the classical Crofton formula, but for Minkowski tensors $\Phi_{k}^{r, 0}$ it takes a sufficiently simple form so that the proof of Theorem 10 carries over. For a convex body $K$ contained in a flat $E \subset \mathbb{R}^{n}$, there are variants of the Minkowski tensors denoted by $\Phi_{j}^{r, s(E)}(K)$. These Minkowski tensors are again tensor valuations of rank $r+s$ in $\mathbb{R}^{n}$, but they are calculated with respect to the support measures of $K$ in $E$; see the beginning of [15, Section 3] for details.

Theorem 12 (Auneau-Cognacq et al. [6, Corollary 4.4]). For $q=1, \ldots, n-1$, $j=1, \ldots, q$ and $r$ a non-negative integer, let $\varphi=\Phi_{n-q+j}^{r, 0}$ be the tensor of rank $r$ with $s=0$ and index $n-q+j$. Then,

$$
\int_{G(n, q)} \varphi_{L}(K \cap L) \nu_{q}(\mathrm{~d} L)=\varphi(K)
$$

$K \in \mathcal{K}^{n}$, where

$$
\varphi_{L}\left(K^{\prime}\right)=\frac{\omega_{n-q+1}}{\omega_{1}} \frac{1}{\alpha_{n, j-1, q-1}} \int_{A(L, q-1)} d(o, E)^{n-q} \Phi_{j-1}^{r, 0(L)}\left(K^{\prime} \cap E\right) \mu_{q-1}(\mathrm{~d} E),
$$

$K^{\prime} \in \mathcal{K}_{L}^{q}$. For $j=q$, (4.1) takes the following explicit form

$$
\varphi_{L}\left(K^{\prime}\right)=\frac{\omega_{n}}{\omega_{q}} \frac{1}{r !} \int_{K^{\prime}} x^{r}\|x\|^{n-q} \mathrm{~d} x,
$$

while for $j=q-1$, (4.1) can equivalently be expressed as

$$
\varphi_{L}\left(K^{\prime}\right)=\frac{\omega_{n}}{\omega_{q}} \frac{1}{r !} \int_{\mathbf{n c}\left(K^{\prime}\right)} x^{r}\|x\|^{n-q} F_{-\frac{1}{2},-\frac{n-q}{2} ; \frac{q-1}{2}}\left(\sin ^{2} \angle(x, u)\right) \Lambda_{q-1}^{L}\left(K^{\prime}, \mathrm{d}(x, u)\right) .
$$

A result of the type (4.1) can also be established for $\Phi_{n-q+j}^{r, 1}$, see [6, Corollary 4.4], but here explicit expressions for $\varphi_{L}$ for $j=q$ and $j=q-1$ are not available.

Surface tensors $\Phi_{k}^{0, s}$ are studied in [24]. In [24, Theorem 3.4], $\Phi_{n-1}^{0, s}(K)$ is expressed for even $s$ as a Crofton-integral with respect to lines $E \in A(n, 1)$, involving an explicitly known tensor $G_{s}(\pi(E))$ of rank $s$. Here, $\pi(E)$ is the line through the origin parallel to $E$. By combining this result with $(3.11), \Phi_{n-1}^{0, s}(K)$ can for even $s$ be expressed as a rotational integral. We get for $q=1, \ldots, n-1$

$$
\Phi_{n-1}^{0, s}(K)=\int_{G(n, q)} \varphi_{L}(K \cap L) \nu_{q}(\mathrm{~d} L)
$$


where

$$
\varphi_{L}\left(K^{\prime}\right)=\frac{\omega_{n-1}}{\omega_{q-1}} \int_{A(L, 1)} d(o, E)^{n-q} G_{s}(\pi(E)) V_{0}(K \cap E) \mu_{1}^{L}(\mathrm{~d} E) .
$$

As is apparent from the discussion above, it is an open problem to express Minkowski tensors with general indices as rotational averages. One possible route to follow for the tensors $\Phi_{k}^{0, s}$ with arbitrary non-negative integer $s$ is to use the recently established kinematic formula [8, Corollary 6.1] for trace-free tensors $\Psi_{k}^{s}$ in combination with the Blaschke-Petkantschin formula (3.11). For $k, l \geq 0, k+l \leq n$ and $n<l+p$, we get

$$
\begin{aligned}
\frac{\omega_{s+k+l}}{\omega_{s+k} \omega_{l}}\left(\begin{array}{c}
k+l \\
k
\end{array}\right) \frac{k l}{k+l}\left[\begin{array}{c}
n \\
l
\end{array}\right]^{-1} \Psi_{k+l}^{s}(K) & =\int_{A(n, n-l)} \Psi_{k}^{s}(K \cap E) \mu_{n-l}(\mathrm{~d} E) \\
& =\int_{G(n, p)} \alpha_{p, k, l}^{s}(K, L) \nu_{p}(\mathrm{~d} L),
\end{aligned}
$$

where

$$
\alpha_{p, k, l}^{s}(K, L)=\frac{\omega_{l}}{\omega_{p-n+l}} \int_{A(L, n-l)} \Psi_{k}^{s}(K \cap E) d(o, E)^{n-p} \mu_{L}(\mathrm{~d} E) .
$$

Combining this with the fact that $\Phi_{k}^{0, s}$ can be expressed in terms of $\Psi_{k}^{0}, \ldots, \Psi_{k}^{s}$ ([8, Proposition 4.16]) it can be seen that any translation invariant Minkowski tensor $\Phi_{k}^{0, s}, 2 \leq k \leq n-1, s \in \mathbb{N}_{0}$, can be written as a non-trivial rotational Crofton integral. To the best of our knowledge, explicit general formulae cannot be found in the literature. It is an open problem to express the more general Minkowski tensors $\Phi_{k}^{r, s}$ as rotational averages.

The situation is much more clear for rotational averages of Minkowski tensors, due to the recent work of Svane ([30]). Using the same techniques as in [18], Theorem 11 can be generalized as follows, where it should be noted that the integrand of the function $Q_{q}$ in (4.4) now depends also on $p_{L} u$ when $s>0$.

Theorem 13 (Svane [30]). Let $q=1, \ldots, n-1, j=0, \ldots, q, r, s$ non-negative integers and let $\left\{\varphi_{L}: L \in G(n, q)\right\}$ be the $\mathrm{SO}(n)$-compatible family given by

$$
\varphi_{L}\left(K^{\prime}\right)=\Phi_{j}^{r, s(L)}\left(K^{\prime}\right)
$$

$K^{\prime} \in \mathcal{K}_{L}^{q}$. Then,

$$
\int_{G(n, q)} \varphi_{L}(K \cap L) \nu_{q}(\mathrm{~d} L)=\varphi(K),
$$

$K \in \mathcal{K}^{n}$, where for $j=q$ and $s=0$

$$
\varphi(K)=\frac{1}{r !} \frac{\omega_{q}}{\omega_{n}} \int_{K} x^{r}\|x\|^{-(n-q)} \mathrm{d} x .
$$

If $K \in \mathcal{K}^{n}$ contains o in its interior, then for $j<q$

$$
\begin{aligned}
\varphi(K)= & \frac{1}{r ! s !} \frac{2 \omega_{q}}{\omega_{n} \omega_{q-j+s}} \int_{\mathbf{n c} K} x^{r}\|x\|^{-(n-q)} \\
& \times \sum_{\substack{I \subset\{1, \ldots, n-1\} \\
|I|=q-j-1}} Q_{q}\left(x, u, A_{I}\right) \frac{\prod_{i \in I} \kappa_{i}(x, u)}{\prod_{i=1}^{n-1} \sqrt{1+\kappa_{i}^{2}(x, u)}} \Lambda_{n-1}(\mathrm{~d}(x, u)),
\end{aligned}
$$


where

$$
Q_{q}\left(x, u, A_{I}\right)=\int_{G(\operatorname{span}\{x\}, q)}\left(p_{L} u\right)^{s} \frac{\left[L, A_{I}\right]^{2}}{\left\|p_{L} u\right\|^{q-j+s}} \nu_{q}^{\operatorname{span}\{x\}}(\mathrm{d} L) .
$$

For $j=q-1$, (4.4) takes the following explicit form

$$
\begin{aligned}
\varphi(K)= & \frac{2}{r ! s ! \omega_{s+1}} \frac{\omega_{q} \omega_{q-1} \omega_{n-q}}{\omega_{n} \omega_{n-1} \omega_{n-2}} \sum_{a+b+c+2 l=s}\left(\begin{array}{c}
s \\
a, b, c, 2 l
\end{array}\right) \frac{\omega_{2 l+n-2}}{\omega_{2 l+1}} \\
& \times \sum_{e+f+t+v=l}\left(\begin{array}{c}
l \\
e, f, t, v
\end{array}\right)(-1)^{f+v+b} 2^{t+1} Q^{e} \\
& \times \int_{\mathbf{n c} K} u^{c+2 f+t} \frac{x^{r+a+b+2 v+t}}{\|x\|^{n-q+a+b+2 v+t}} g\left(\sin ^{2} \angle(x, u)\right) \Lambda_{n-1}(K ; \mathrm{d}(x, u)),
\end{aligned}
$$

where $Q \in \mathbb{T}^{2}$ is the metric tensor and

$$
g\left(\alpha^{2}\right)=\frac{\omega_{n-1+2 b+2 c+4 l}}{\omega_{q-1+2 b+2 c+2 l} \omega_{n-q+2 l}} \alpha^{2 e}\left(1-\alpha^{2}\right)^{\frac{a+b+t}{2}} F_{\frac{s-1}{2}, \frac{n-q}{2}+l ; \frac{n-1}{2}+b+c+2 l}\left(\alpha^{2}\right) .
$$

We finally mention that a recently derived kinematic Crofton formula for area measures [14] can also be combined with the Blaschke-Petkantschin formula (3.11) in order to obtain a rotational Crofton-type representation of the surface area measure $S_{k}(K, \cdot)$ of $K$ with index $2 \leq k \leq n-1$.

\section{Uniqueness of the measurement function}

Let $K \in \mathcal{K}^{n}$ and $q \in\{1, \ldots, n-1\}$ be given. The rotational Crofton formulae in Section 4 all read

$$
\int_{G(n, q)} \varphi_{L}(K \cap L) \nu_{q}(\mathrm{~d} L)=\varphi(K)
$$

where $\varphi$ is some tensor valued valuation and the functionals $\varphi_{L}$ are tensor valued valuations on $\mathcal{K}_{L}^{q}$ for all $L \in G(n, q)$. As $\varphi_{L}$ is the quantity we have to measure in order to obtain a desired isotropic average, we refer to $\varphi_{L}$ as the measurement function. In [10] it was asked if this measurement function is unique under appropriate additional assumptions when the right hand side of (5.1) is an intrinsic volume of $K$. This question was motivated by the observation that two apparently different measurement functions that satisfy (5.1) with $\varphi=V_{n}$ actually coincide. In fact, also the following result on surface area estimation appears to support uniqueness of the measurement function. Theorem 10 implies that (5.1) with $\varphi=V_{n-1}$ holds with $\varphi_{L}$ given by (3.10). About a decade before Theorem 10 was established, a BlaschkePetkantschin formula was used in [16, Section 5.6] to show that the apparently different measurement function

$$
\varphi_{L}\left(K^{\prime}\right)=\frac{1}{2} \frac{\omega_{n}}{\omega_{q}} \int_{\mathbb{S}^{n-1} \cap L} \rho_{K}^{n-1}(u) \frac{1}{\cos \gamma_{L}(u)} F_{-\frac{1}{2},-\frac{n-q}{2}, \frac{q-1}{2}}\left(\sin ^{2} \gamma_{L}(u)\right) \mathrm{d} u,
$$


$K^{\prime} \in \mathcal{K}_{L}^{q}$, also satisfies (5.1) if $o \in \operatorname{int} K$. Here $\gamma_{L}(u)$ is the angle between $u$ and the (almost everywhere unique) outer unit normal in $L$ of $K^{\prime}$ at its boundary point $u \rho_{K^{\prime}}(u)$. A closer examination reveals that the measurement functions (3.10) and (5.2) actually coincide when $q=2$; see [11] for a proof in the case of strictly convex and smooth $K \subset \mathbb{R}^{3}$ and [31] for the general case.

Using the linearity of the integral, the original uniqueness question can equivalently be rephrased by asking under what conditions

$$
\int_{G(n, q)} \varphi_{L}(K \cap L) \nu_{q}(\mathrm{~d} L)=0
$$

implies that all measurement functions $\varphi_{L}$ are vanishing.

In contrast to the convex case, the corresponding question for measurement functions on $L^{n}$-stars is not difficult: We have already noted after Corollary 4 that an $\mathrm{SO}(n)$-compatible family $\left\{\varphi_{L}: L \in G(n, q)\right\}$ of continuous valuations is uniquely determined when $\varphi_{L}$ is known on all balls in $L$, so (5.3) implies that all $\varphi_{L}$ vanish. When $q=1$ any member of an $\operatorname{SO}(n)$-compatible family $\left\{\varphi_{L}: L \in G(n, q)\right\}$ of functionals $\varphi_{L}: \mathcal{S}_{L}^{n} \rightarrow \mathbb{R}$ (without any further assumptions) must vanish when (5.3) holds. In fact, one only has to show that $\varphi_{L}$ vanishes on all line-segments in $L$ that contain the origin. However, this is a direct consequence of (5.3) applied to the sets

$$
K=r B^{n} \cup\left\{x \in R B^{n}:\langle x, w\rangle \geq 0\right\},
$$

$0 \leq r \leq R, w \in \mathbb{S}^{n-1}$, and the $\mathrm{SO}(n)$-compatibility.

The question under what conditions (5.3) determines $\varphi_{L}$ in the convex case is widely open apart from the following result on one-dimensional sections.

Theorem 14. Let $\left\{\varphi_{L}: L \in G(n, 1)\right\}$ be an $\mathrm{SO}(n)$-compatible family of functionals $\varphi_{L}$ on $\mathcal{K}_{L}^{n}$. Then (5.3) implies that $\varphi_{L}=0$ for all $L \in \mathcal{L}_{1}^{n}$.

The proof uses the convex hull $K_{1}$ of $K$ in (5.4) and the intersection $K_{2}$ of all closed supporting half spaces of $K_{1}$ that contain a point of $\left\{x \in R B^{n}:\langle x, w\rangle \geq 0\right\}$ in their boundaries. An explicit calculation and comparison of (5.3) with $K_{1}$ and $K_{2}$ replacing $K$ then yields the assertion.

\section{Principal rotational formulae}

A principal rotational formula for Minkowski tensors may involve integrals of the form

$$
\int_{\mathrm{SO}(n)} \Phi_{k}^{r, s}(K \cap \vartheta M) \nu(\mathrm{d} \vartheta)
$$

$k=0, \ldots, n, r, s \in \mathbb{N}_{0}$, where $K, M \in \mathcal{K}^{n}$ and $\nu$ is the unique rotation invariant probability measure on $\mathrm{SO}(n)$. In local stereology, principal rotational formulae are used in cases where an unknown spatial structure $K$ is studied via the intersection with a randomly rotated set $M$. In such applications, $M$ is a known 'sampling window' constructed by the observer. 
In this section, we consider principal rotational formulae for general Minkowski tensors. It turns out that local Minkowski tensors are an important tool in the derivation of such formulae. For $K \in \mathcal{K}^{n}, r, s$ non-negative integers and $k=0, \ldots, n-1$, the local Minkowski tensors are defined by

$$
\Phi_{k}^{r, s}(K, \psi):=\frac{\omega_{n-k}}{r ! s ! \omega_{n-k+s}} \int_{\mathbf{n c} K} \psi(x, u) x^{r} u^{s} \Lambda_{k}(K, \mathrm{~d}(x, u))
$$

and

$$
\Phi_{n}^{r, 0}(K, \phi):=\frac{1}{r !} \int_{K} \phi(x) x^{r} \mathrm{~d} x
$$

where $\psi$ and $\phi$ are non-negative measurable functions on $\mathbb{R}^{n} \times \mathbb{S}^{n-1}$ and $\mathbb{R}^{n}$, respectively. The classical Minkowski tensors are obtained in (6.1) and (6.2) by choosing the functions $\psi$ and $\phi$ identically equal to 1 . We remark for later use that the rotation group acts on the corresponding function spaces in the natural way: for $\vartheta \in \mathrm{SO}(n)$, let $(\vartheta \psi)(x, u)=\psi\left(\vartheta^{-1} x, \vartheta^{-1} u\right)$ and $(\vartheta \phi)(x)=\phi\left(\vartheta^{-1} x\right), x \in \mathbb{R}^{n}, u \in \mathbb{S}^{n-1}$. We define the rotational average

$$
\bar{\psi}(x, u)=\int_{\mathrm{SO}(n)} \psi(\vartheta x, \vartheta u) \nu(\mathrm{d} \vartheta)
$$

and likewise for $\bar{\phi}$. The same definition can also be applied to functions $\psi$ and $\phi$ with values in $\mathbb{T}^{p}$.

A simple application of Tonelli's theorem yields a principal rotational formula for local Minkowski tensors.

Proposition 15. Let $\psi$ and $\phi$ be non-negative measurable functions on $\mathbb{R}^{n} \times \mathbb{S}^{n-1}$ and $\mathbb{R}^{n}$, respectively. Then, for $K \in \mathcal{K}^{n}, r, s$ non-negative integers and $k=0, \ldots$, $n-1$,

$$
\int_{\mathrm{SO}(n)} \Phi_{n}^{r, 0}(K, \vartheta \phi) \nu(\mathrm{d} \vartheta)=\Phi_{n}^{r, 0}(K, \bar{\phi})
$$

and

$$
\int_{\mathrm{SO}(n)} \Phi_{k}^{r, s}(K, \vartheta \psi) \nu(\mathrm{d} \vartheta)=\Phi_{k}^{r, s}(K, \bar{\psi})
$$

As a consequence of Proposition 15, we have the following principal rotational formula for local Minkowski tensors. We slightly abuse notation using $\mathbf{1}_{A}(x, u)=$ $\mathbf{1}_{A}(x)$ for the indicator function of a set $A \subset \mathbb{R}^{n}$ and $\phi_{M}(x, u)=\phi_{M}(x)$ for the function $\phi_{M}$ defined below, where $(x, u) \in \mathbb{R}^{n} \times \mathbb{S}^{n-1}$.

Theorem 16. Let $K, M \in \mathcal{K}^{n}$ and

$$
\phi_{M}(x)=\frac{\mathcal{H}^{n-1}\left(\operatorname{int} M \cap\|x\| \mathbb{S}^{n-1}\right)}{\mathcal{H}^{n-1}\left(\|x\| \mathbb{S}^{n-1}\right)},
$$

if $x \in \mathbb{R}^{n} \backslash\{o\}$, and $\phi_{M}(o)=\mathbf{1}_{\mathrm{int} M}(o)$. Then, for any non-negative integer $r$ we have

$$
\int_{\mathrm{SO}(n)} \Phi_{n}^{r, 0}(K \cap \vartheta M) \nu(\mathrm{d} \vartheta)=\Phi_{n}^{r, 0}\left(K, \phi_{M}\right)
$$


If, in addition, $k=0, \ldots, n-1$, and $s \in \mathbb{N}_{0}$ we have

$$
\int_{\mathrm{SO}(n)} \Phi_{k}^{r, s}\left(K \cap \vartheta M, \vartheta \mathbf{1}_{\mathrm{int} M}\right) \nu(\mathrm{d} \vartheta)=\Phi_{k}^{r, s}\left(K, \phi_{M}\right) .
$$

When $k=n-1$ and $\mathcal{H}^{n-1}(\mathrm{bd} K \cap \vartheta \mathrm{bd} M)=0$ for almost all $\vartheta \in \mathrm{SO}(n)$, this implies

$$
\begin{aligned}
\int_{\mathrm{SO}(n)} & \Phi_{n-1}^{r, s}(K \cap \vartheta M) \nu(\mathrm{d} \vartheta) \\
= & \Phi_{n-1}^{r, s}\left(K, \phi_{M}\right)+\frac{1}{r ! s !} \frac{2}{\omega_{s+1}} \int_{\mathbf{n c} M} \overline{\mathbf{1}_{\operatorname{int} K} x^{r} u^{s}} \Lambda_{n-1}(M, \mathrm{~d}(x, u)) .
\end{aligned}
$$

Proof. As $\overline{\mathbf{1}_{\text {int } M}}=\phi_{M}$ and

$$
\Phi_{n}^{r, 0}(K \cap \vartheta M)=\Phi_{n}^{r, 0}\left(K, \vartheta \mathbf{1}_{\text {int } M}\right),
$$

(6.3) follows directly from Proposition 15. Support measures are locally defined, so $\Lambda_{k}(K \cap \vartheta M, \eta)=\Lambda_{k}(K, \eta)$ for the open set $\eta=(\operatorname{int} M) \times \mathbb{S}^{n-1}$. This implies

$$
\Phi_{k}^{r, s}\left(K \cap \vartheta M, \vartheta \mathbf{1}_{\text {int } M}\right)=\Phi_{k}^{r, s}\left(K, \vartheta \mathbf{1}_{\text {int } M}\right)
$$

and Proposition 15 yields (6.4). To show (6.5) an application of the facts that support measures are locally defined together with the additional assumption yields

$$
\begin{aligned}
\Lambda_{n-1}(K \cap \vartheta M, \cdot)= & \Lambda_{n-1}\left(K, \cdot \cap\left(\vartheta(\operatorname{int} M) \times \mathbb{S}^{n-1}\right)\right) \\
& +\Lambda_{n-1}\left(\vartheta M, \cdot \cap\left((\operatorname{int} K) \times \mathbb{S}^{n-1}\right)\right)
\end{aligned}
$$

for almost all $\vartheta$. Integrating $x^{r} u^{s}$ with this measure, applying (6.4) and using again the fact that support measures are locally defined to simplify the second term, yields (6.5).

Proposition 15 may also be used to derive a principal rotational formula where Minkowski tensors are expressed as rotational averages. The result is given in the theorem below.

Theorem 17. Let $K, M \in \mathcal{K}^{n}$. Suppose that $M$ is chosen such that $o \in \operatorname{int} M$ and that

$$
\mathcal{H}^{n-1}\left(\text { int } M \cap\|x\| \mathbb{S}^{n-1}\right)>0
$$

for all $o \neq x \in K$. Let

$$
\phi_{M}^{\circ}(x)=\frac{\mathcal{H}^{n-1}\left(\|x\| \mathbb{S}^{n-1}\right)}{\mathcal{H}^{n-1}\left(\operatorname{int} M \cap\|x\| \mathbb{S}^{n-1}\right)} \mathbf{1}_{\text {int } M}(x),
$$

if $\mathcal{H}^{n-1}$ (int $\left.M \cap\|x\| \mathbb{S}^{n-1}\right)>0$, and $\phi_{M}^{\circ}(x)=0$, otherwise. Then, for $r, s$ non-negative integers and $k=0, \ldots, n-1$,

$$
\int_{\mathrm{SO}(n)} \Phi_{k}^{r, s}\left(K \cap M, \vartheta \phi_{M}^{\circ}\right) \nu(\mathrm{d} \vartheta)=\Phi_{k}^{r, s}(K)
$$

and

$$
\int_{\mathrm{SO}(n)} \Phi_{n}^{r, 0}\left(K \cap M, \vartheta \phi_{M}^{\circ}\right) \nu(\mathrm{d} \vartheta)=\Phi_{n}^{r, 0}(K)
$$


The theorem follows from Proposition 15 as $\overline{\phi_{M}^{\circ}}(x)=1$ for $x \in K$.

In order to use Theorem 17 for estimating $\Phi_{k}^{r, s}(K)$ from an observation in $K \cap \vartheta M$, where $\vartheta$ is a random rotation, requires to determine the weight function $\phi_{M}^{\circ}$ yielding a Horvitz-Thompson-type correction. This is possible when $\vartheta M$ is known which is often the case in optical microscopy, see e.g. [29].

But from a basic science point of view, it is important to develop principal rotational formulae of the type (6.3) and (6.5) with integrands only depending on $K \cap \vartheta M$ without any further knowledge. To the best of our knowledge, this is an open problem in rotational integral geometry for the measurement function $\Phi_{k}^{r, s}$ with $k<n-1$.

\section{Local stereology applications}

The aim of local stereology ([16]) is the estimation of quantitative parameters (volume, surface area, Minkowski tensors, ... ) of spatial structures from sections through fixed points, called reference points.

Using a rotational Crofton formula, local stereological estimators of Minkowski tensors $\Phi_{k}^{r, s}(K)$ have recently been derived ([19]), based on measurements on random sections passing through a fixed point of $K$. More specifically, such local estimators are available for (i) $s=0,1$ and $r, k$ arbitrary and for (ii) $r=0, s$ even and $k=n-1$. In (i), the rotational Crofton formula presented in [6, Corollary 4.4] is used while (ii) follows by combining [24, Theorem 3.4] with the Blaschke-Petkantschin formula (3.11). The details were given in Section 4. The most common stereological application of rotational Crofton formula is the estimation of intrinsic volumes $(r=$ $s=0$ ). For volume and surface area, that is when $k=n$ or $k=n-1$, different forms of measurement functions have been suggested. In [31] several surface area estimators are discussed and a measurement function based on Morse theory is established. The works of Cruz-Orive and Gual-Arnau on this subject are summarized in the recent paper [12].

Alternatively, measurements for local estimation of $\Phi_{k}^{r, s}(K)$ may be performed on the intersection $K \cap \mathbf{M}$ of $K$ with a randomly rotated convex body $\mathbf{M}$. Here, a principal rotational formula is used; see Section 6 .

In this section, we will investigate to what extent these results can be transferred to particle processes. Let $X$ be a particle process of full-dimensional convex particles in $\mathbb{R}^{n}$ that we represent as a stationary marked point process. The marked point process is given by

$$
\{[x(K) ; K-x(K)]: K \in X\},
$$

where $x(K) \in K$ is a reference point associated to each particle $K \in X$ while the mark $K-x(K)$ is the particle translated such that its reference point is the origin $o$. The particle mark distribution is denoted by $\mathbb{Q}$. We let $\mathbf{K}_{0}$ be a random convex set with distribution $\mathbb{Q}$. We may regard $\mathbf{K}_{0}$ as a randomly chosen particle or a typical particle with $o$ put at its reference point.

Inference about the distribution of $\Phi_{k}^{r, s}\left(\mathbf{K}_{0}\right)$ may be based on a sample of particles, collected as those particles with reference point in a sampling window. More 
specifically, we consider a sample of the form

$$
\{K \in X: x(K) \in W\}
$$

where $W \in \mathcal{B}\left(\mathbb{R}^{n}\right)$ is a full-dimensional sampling window with $0<\lambda_{n}(W)<\infty$. The distribution of $\Phi_{k}^{r, s}\left(\mathbf{K}_{0}\right)$ may be studied via the empirical distribution of

$$
\left\{\Phi_{k}^{r, s}(K-x(K)): K \in X, x(K) \in W\right\} .
$$

If complete access to the sampled particles is not possible, the distribution of $\Phi_{k}^{r, s}\left(\mathbf{K}_{0}\right)$ may still be studied via $(7.2)$ if a precise estimate $\widehat{\Phi}_{k}^{r, s}(K-x(K))$ of $\Phi_{k}^{r, s}(K-x(K))$ is available, e.g. from replicated local sectioning of $K-x(K)$.

We will now discuss the situation where such precise estimates are not available. For this discussion, it turns out to be useful to consider the following $n+1$ probability measures $P_{X k}, k=0, \ldots, n$, associated to the particle process $X$. The probability measure $P_{X n}$ is concentrated on $\mathbb{R}^{n}$ and is absolutely continuous with respect to the Lebesgue measure $\lambda_{n}$ with probability density

$$
f_{\mathbf{K}_{0}}(x)=P\left(x \in \mathbf{K}_{0}\right) / \mathbb{E} \lambda_{n}\left(\mathbf{K}_{0}\right), \quad x \in \mathbb{R}^{n},
$$

called the cover density. The density $f_{\mathbf{K}_{0}}$ may be envisaged as a kind of probability cloud. If $\mathbf{K}_{0}$ is deterministic, then $f_{\mathbf{K}_{0}}$ is proportional to $\mathbf{1}_{\mathbf{K}_{0}}$, so $\mathbf{K}_{0}$ can be reconstructed from $f_{\mathbf{K}_{0}}$. If $\mathbb{Q}$ is invariant under rotations, then $f_{\mathbf{K}_{0}}$ is also rotation invariant.

The remaining probability measures $P_{X k}, k=0, \ldots, n-1$, are concentrated on $\mathbb{R}^{n} \times \mathbb{S}^{n-1}$ and are normalized versions of the mean support measures

$$
P_{X k}(A)=\frac{\mathbb{E} \Lambda_{k}\left(\mathbf{K}_{0}, A\right)}{\mathbb{E} \Lambda_{k}\left(\mathbf{K}_{0}, \mathbb{R}^{n} \times \mathbb{S}^{n-1}\right)}, \quad A \in \mathcal{B}\left(\mathbb{R}^{n} \times \mathbb{S}^{n-1}\right)
$$

The probability measures $P_{X k}, 0 \leq k \leq n-1$, contain information about the probabilistic properties of the boundary of $\mathbf{K}_{0}$. As an example, $P_{X(n-1)}\left(\mathbb{R}^{n} \times \cdot\right)$ is proportional to the surface area measure of the so-called Blaschke body $B(X)$ of the particle process, see $[28$, p. 149$]$. If $\mathbb{Q}$ is invariant under rotations, then $B(X)$ is a ball.

The theorem below shows that for particle processes, normalized mean Minkowski tensors determine the moments of arbitrary order in the distributions $P_{X k}$, $k=0, \ldots, n$.

Theorem 18. Let $X$ be a stationary particle process of full-dimensional convex particles in $\mathbb{R}^{n}$ with particle mark distribution $\mathbb{Q}$. Let $\mathbf{K}_{0}$ be a random convex set with distribution $\mathbb{Q}$. Then, for non-negative integers $r, s$ and $k=0, \ldots, n-1$

$$
\frac{\mathbb{E} \Phi_{k}^{r, s}\left(\mathbf{K}_{0}\right)}{\mathbb{E} \Phi_{k}^{0,0}\left(\mathbf{K}_{0}\right)}=\frac{\omega_{n-k}}{r ! s ! \omega_{n-k+s}} \int_{\mathbb{R}^{n} \times \mathbb{S}^{n-1}} x^{r} u^{s} P_{X k}(\mathrm{~d}(x, u)) .
$$

For $k=n$, we get

$$
\frac{\mathbb{E} \Phi_{n}^{r, 0}\left(\mathbf{K}_{0}\right)}{\mathbb{E} \Phi_{n}^{0,0}\left(\mathbf{K}_{0}\right)}=\frac{1}{r !} \int_{\mathbb{R}^{n}} x^{r} f_{\mathbf{K}_{0}}(x) \lambda_{n}(\mathrm{~d} x) .
$$


For $k=n-1$ and $r=0$ we have the following connection to the surface area measure of the Blaschke body:

$$
\frac{\mathbb{E} \Phi_{n-1}^{0, s}\left(\mathbf{K}_{0}\right)}{\mathbb{E} \Phi_{n-1}^{0,0}\left(\mathbf{K}_{0}\right)}=\frac{1}{s ! \omega_{s+1}} \int_{\mathbb{S}^{n-1}} u^{s} \frac{S_{n-1}(B(X), \mathrm{d} u)}{V_{n-1}(B(X))} .
$$

Let us now return to the problem of drawing inference about the distribution of $\Phi_{k}^{r, s}\left(\mathbf{K}_{0}\right)$ from a sample of particles. Using Campbell's theorem for marked point processes, we have

$$
\frac{\mathbb{E} \sum_{K \in X, x(K) \in W} \Phi_{k}^{r, s}(K-x(K))}{\mathbb{E} \sum_{K \in X, x(K) \in W} \Phi_{k}^{0,0}(K-x(K))}=\frac{\mathbb{E} \Phi_{k}^{r, s}\left(\mathbf{K}_{0}\right)}{\mathbb{E} \Phi_{k}^{0,0}\left(\mathbf{K}_{0}\right)} .
$$

Combining this result with Theorem 18, it follows under weak assumptions about the particle process that

$$
\frac{r ! s ! \omega_{n-k+s}}{\omega_{n-k}} \frac{\sum_{K \in X, x(K) \in W} \Phi_{k}^{r, s}(K-x(K))}{\sum_{K \in X, x(K) \in W} \Phi_{k}^{0,0}(K-x(K))}
$$

is a consistent (in a probabilistic sense) estimator of the moment of order $(r, s)$ of $P_{X k}$, also in the case where $\Phi_{k}^{r, s}(K-x(K))$ is substituted with an unbiased estimator $\widehat{\Phi}_{k}^{r, s}(K-x(K))$, subject to non-negligable variability. For instance, consistency follows in an expanding window regime if the particle process is ergodic, see $[9$, Corollary 12.2.V].

These ideas have been pursued in detail in [26, 33] for volume tensors and the resulting methods have been implemented in optical microscopy. For a sampled particle $K$, the volume tensor $\Phi_{n}^{r, 0}(K-x(K))$ is here unbiasedly estimated using a local stereological design, involving measurements from the central part of $K$.

The design used in $[26,33]$ is a so-called vertical slice design. Let us consider a slice of the form $T=L+B(o, t)$ where $L \in G(M, q), q>1$, is a $q$-dimensional linear subspace containing a fixed line $M \in G(n, 1)$ passing through $o$ and $t>0$ is the thickness of the slice. The line $M$ is called the vertical axis. The set of such slices is denoted $T(n, q, M)$. We let $\rho_{q}^{M}$ denote the unique probability measure on $T(n, q, M)$, invariant under rotations that keep $M$ fixed.

The unbiased estimator of $\Phi_{n}^{r, 0}(K-x(K))$ is obtained by replacing $K$ by $K-x(K)$ in the lemma below.

Lemma 19. Let $\mathbf{T}$ be a random vertical slice with distribution $\rho_{q}^{M}$. Let $K \in \mathcal{K}^{n}$ be a fixed convex set and $G_{a, b}$ the distribution function of the Beta distribution with parameters $(a / 2, b / 2)$. Then,

$$
\widehat{\Phi}_{n}^{r, 0}(K ; \mathbf{T})=\frac{1}{r !} \int_{K \cap \mathbf{T}} x^{r} G_{n-q, q-1}\left(t^{2} /\left\|p_{M^{\perp}}(x)\right\|^{2}\right)^{-1} \lambda_{n}(\mathrm{~d} x)
$$

is an unbiased estimator of $\Phi_{n}^{r, 0}(K)$.

The lemma is a direct consequence of [16, Proposition 6.3].

Combining Lemma 19 with Theorem 18 and (7.3), we obtain the following result. 
Theorem 20. Let $W \in \mathcal{B}\left(\mathbb{R}^{n}\right)$ with $0<\lambda_{n}(W)<\infty$. Let $X$ be a stationary particle process of convex particles in $\mathbb{R}^{n}$ with particle mark distribution $\mathbb{Q}$. Let $\mathbf{K}_{0}$ be a random convex set with distribution $\mathbb{Q}$. Finally, let $\mathbf{T}$ be a random vertical slice, independent of the particle process $X$, with distribution $\rho_{q}^{M}$. Then, for any non-negative integer $r$,

$$
\frac{\mathbb{E} \sum_{K \in X, x(K) \in W} \widehat{\Phi}_{n}^{r, 0}(K-x(K) ; \mathbf{T})}{\mathbb{E} \sum_{K \in X, x(K) \in W} \widehat{\Phi}_{n}^{0,0}(K-x(K) ; \mathbf{T})}=\frac{1}{r !} \int_{\mathbb{R}^{n}} x^{r} f_{\mathbf{K}_{0}}(x) \lambda_{n}(\mathrm{~d} x),
$$

where $\widehat{\Phi}_{n}^{r, 0}$ is given in Lemma 19.

If the particle mark distribution $\mathbb{Q}$ is invariant under rotations that keep the vertical axis fixed, then it is not needed to randomize the slice.

\section{Acknowledgements}

This research was supported by Centre for Stochastic Geometry and Advanced Bioimaging, funded by a grant from the Villum Foundation.

\section{References}

[1] Alesker, S.: Continuous rotation invariant valuations on convex sets. Ann. Math., 149, 977-1005 (1999)

[2] Alesker, S.: Theory of valuations on manifolds, I. Linear spaces. Israel J. Math. 156, 311-399 (2006)

[3] Alesker, S.: Erratum: Rotation invariant valuations on convex sets. Ann. Math. 166, 947-948 (2007)

[4] Auneau, J., Rataj, J., Jensen, E.B.V.: Closed form of the rotational Crofton formula. Math. Nachr. 285, 164-180 (2012)

[5] Auneau, J., Jensen, E.B.V.: Expressing intrinsic volumes as rotational integrals. Adv. Appl. Math. 45, 1-11 (2010)

[6] Auneau-Cognacq, J., Ziegel, J., Jensen, E.B.V.: Rotational integral geometry of tensor valuations. Adv. Appl. Math. 50, 429-444 (2013)

[7] Beals, R., Wong, R.: Special Functions. Cambridge University Press, Cambridge (2010)

[8] Bernig, A., Hug, D.: Kinematic formulas for tensor valuations. J. Reine. Angew. Math., to appear (2015)

[9] Daley, D.J., Vere-Jones, D.: An Introduction to the Theory of Point Processes. Vol. II. General Theory and Structure. (2nd ed.) Springer, New York (2008)

[10] Gual-Arnau, X., Cruz-Orive, L.M., Nuno-Ballesteros, J.J.: A new rotational integral formula for intrinsic volumes in space forms. Adv. Appl. Math. 44, 298-308 (2010) 
[11] Cruz-Orive, L.M.: Uniqueness properties of the invariator, leading to simple computations. Image Anal. Stereol. 31, 89-98 (2012)

[12] Cruz-Orive, L.M., Gual-Arnau, X.: The invariator design: an update. Image Anal. Stereol. 34, 147-159 (2015)

[13] Gardner, R.J.: Geometric Tomography. (2nd ed.) Cambridge University Press, Cambridge (2006)

[14] Goodey, P., Hug, D., Weil, W.: Kinematic formulas for area measures. preprint arXiv:1507.03353 (2015)

[15] Hug, D., Schneider, R., Schuster, R.: Integral geometry of tensor valuations. Adv. Appl. Math. 41, 482-509 (2008)

[16] Jensen, E.B.V.: Local Stereology. World Scientific Publishing, Singapore (1998)

[17] Jensen, E.B.V., Rasmusson, A.: Rotational integral geometry and local stereology âĂŞ with a view to image analysis. In: Schmidt, V. (ed.) Stochastic Geometry, Spatial Statistics and Random Fields. Models and Algorithms. Lecture Notes in Mathematics 2120, pp. 233-255. Springer, Heidelberg (2015)

[18] Jensen, E.B.V., Rataj, J.: A rotational integral formula for intrinsic volumes. Adv. Appl. Math. 41, 530-560 (2008)

[19] Jensen, E.B.V., Ziegel, J.F.: Local stereology of tensors of convex bodies. Methodol. Comput. Appl. 16, 263-282 (2014)

[20] Khovanskii, A.G., Pukhlikov, A.V.: Finitely additive measures on virtual polyhedra (Russian). Algebra i Analiz 4 161-85 (1992); translation in St. Petersburg Math. J. 4 337-356 (1993)

[21] Kiderlen, M.: Introduction into integral geometry and stereology. In: Spodarev, E. (ed.) Stochastic Geometry, Spatial Statistics and Random Fields, Lecture notes in Mathematics 2068, pp. 21-48. Springer, Heidelberg (2013)

[22] Klain, D.A.: Star valuations and dual mixed volumes. Adv. Math. 121 80-108 (1996)

[23] Klain, D.A.: Invariant valuations on star-shaped sets. Adv. Math. 125, 95-113 (1997)

[24] Kousholt, A., Kiderlen, M., Hug, D.: Surface tensor estimation from linear sections. CSGB Research Report 14-7 (2014). Submitted.

[25] Lutwak, E.: Mean dual and harmonic cross-sectional measures. Ann. Mat. Pura Appl. 119, 139-148 (1979)

[26] Rafati, A.H., Ziegel, J.F., Nyengaard J.R., Jensen E.B.V.: Stereological estimation of particle shape and orientation from volume tensors. CSGB Research Report 15-12 (2015). Submitted.

[27] Schneider, R.: Convex Bodies: The Brunn-Minkowski Theory. (2nd ed.) Cambridge University Press, Cambridge (2014)

[28] Schneider, R., Weil, W.: Stochastic and Integral Geometry. Springer, Heidelberg (2008) 
[29] Stark, A.K., Gundersen, H.J.G., Gardi, J.E., Pakkenberg, B., Hahn, U.: The saucor, a new stereological tool for analysing the spatial distribution of cells, exemplified by human neocortical neurons and glial cells. J. Microsc. 242, 132-147 (2011)

[30] Svane, A.M.: Rotational integrals of Minkowski tensors. CSGB Research Report 15-x (2015).

[31] Thórisdóttir, Ó., Kiderlen, M.: The invariator principle in convex geometry. Adv. Appl. Math. 58, 63-87 (2014)

[32] Villanueva, I.: Radial continuous rotation invariant valuations on star bodies. preprint arXiv:1503.06064 (2015)

[33] Ziegel, J.F., Nyengaard, J.R., Jensen, E.B.V.: Estimating particle shape and orientation using volume tensors. Scand. J. Stat. 42, 813-831 (2015) 